\title{
Pharmaceutical Cocrystals: An Overview
}

\author{
S. KUMAR AND A. NANDA* \\ Department of Pharmaceutical Sciences, Maharshi Dayanand University, Rohtak-124 001, India
}

Kumar and Nanda: Pharmaceutical Cocrystals: An Overview

\begin{abstract}
Poor aqueous solubility and low oral bioavailability of an active pharmaceutical ingredient are the major constraints during the development of new product. Various approaches have been used for enhancement of solubility of poorly aqueous soluble drugs, but success of these approaches depends on physical and chemical nature of molecules being developed. Cocrystallization of drug substances offers a great opportunity for the development of new drug products with superior physicochemical such as melting point, tabletability, solubility, stability, bioavailability and permeability, while preserving the pharmacological properties of the active pharmaceutical ingredient. Cocrystals are multicomponent systems in which two components, an active pharmaceutical ingredient and a coformer were present in stoichiometric ratio and bonded together with non-covalent interactions in the crystal lattice. This review article presents a systematic overview of pharmaceutical cocrystals. Differences between cocrystals with salts, solvates and hydrates are summarized along with the advantages of cocrystals with examples. The theoretical parameters underlying the selection of coformers and screening of cocrystals have been summarized and different methods of cocrystal formation and evaluation have been explained.
\end{abstract}

Key words: Pharmaceutical cocrystals, cocrystallization, solubility, stability, bioavailability, supramolecular synthons

In the last few years, a large number of drugs have been discovered with low aqueous solubility. Among these recently discovered drugs, about $60-70 \%$ of the compounds are related to the BCS Class II (low solubility/high permeability) and IV (low solubility/ low permeability ${ }^{[1,2]}$. Many active pharmaceutical ingredients (APIs) have not been developed in formulations due to low aqueous solubility, which causes low bioavailability of drugs ${ }^{[3]}$. The gastrointestinal tract has different $\mathrm{pH}$ in different parts, so drugs when given by oral route have different solubility in gastrointestinal fluids at different $\mathrm{pH}$, often leading to nonlinear and variable absorption and efficacy and safety of drugs cannot be evaluated properly. That is why limited solubility of drugs is a major challenge in development of oral dosage forms ${ }^{[4]}$.

Researchers have developed various approaches to enhance the solubility of drugs, which lead to improvement in the bioavailability. Size reduction, solid dispersion, complexation, salt formation, nanoparticles, self-emulsifying drug delivery system (SEDDS), addition of co-solvents, nano-suspension and emulsion and cocrystal formation. are some of the approaches used for the solubility enhancement of poorly water-soluble drugs. Each technique has its own merits and demerits and certain factors (such as properties of API, nature of selected excipients, method used for development and nature of dosage form) should be kept in mind for the selection of technique ${ }^{[5]}$.

Amongst all these techniques, cocrystals approach is unique, in that it does not affect the pharmacological properties of the drug, but it may improve the drugs' bioavailability and also improve several of its physicochemical characteristics, such as melting point, tabletability, solubility, stability, bioavailability and permeability.

\section{COCRYSTALS}

The term "cocrystal" and design rules of hydrogen bonding of an organic cocrystal were first reported by $E t t e{ }^{[6,7]}$. Desiraju was the first who gave the

This is an open access article distributed under the terms of the Creative Commons Attribution-NonCommercial-ShareAlike 3.0 License, which allows others to remix, tweak, and build upon the work non-commercially, as long as the author is credited and the new creations are licensed under the identical terms

Accepted 21 September 2017

Revised 24 March 2017

Received 06 October 2016

Indian J Pharm Sci 2017;79(6):858-871 
supramolecular synthon concept of hydrogen bond formation in the crystal structures ${ }^{[8]}$. In 2004, pharmaceutical cocrystals were described as a distinct class of novel, crystalline materials which could alter the physicochemical properties of APIs and this was the beginning of the new era in crystal engineering and cocrystal formation ${ }^{[9]}$. Duggirala and coworkers classified the cocrystals into molecular and ionic depending on the type of coformers ${ }^{[10]}$. In molecular cocrystals, neutral or non-ionized coformers are present in a stoichiometric ratio and mostly reported cocrystals come in this category. Ionic cocrystals were contained ionized coformers in a stoichiometric ratio and formed by charge assisted hydrogen bonds and/or coordination bonds ${ }^{[11]}$. Pharmaceutical cocrystals have been defined as cocrystals, which contained an API as one component and another component as a coformer in a stoichiometric ratio ${ }^{[12]}$.

In literature, researchers have defined the cocrystals in various definitions ${ }^{[13-16]}$. The generally accepted definition of cocrystals was proposed by 46 scientists during the Indo-US Bilateral Meeting sponsored by the Indo-US Science and Technology Forum titled "The Evolving Role of Solid State Chemistry in Pharmaceutical Science", which was organized in Delhi, India in 2012. Researchers proposed a broad definition of cocrystal that was consistent with the scientific literature. Cocrystals are solids that are crystalline single-phase materials composed of two or more different molecular and/or ionic compounds generally in a stoichiometric ratio which are neither solvates nor simple salts ${ }^{[17]}$. In 2013, USFDA proposed a brief definition of cocrystal in the draft guidance as "solids that are crystalline materials composed of two or more molecules in the same crystal lattice"[18].

\section{Difference between cocrystals, salt, solvates and hydrates:}

USFDA defined the cocrystal, salt and polymorphs in the draft guidance. The polymorphs are defined as the compounds which are present in different crystalline forms such as solvates or hydrates (also known as pseudopolymorphs) and amorphous forms. Polymorphs have different lattice arrangement and also, they have different physicochemical properties due to their crystal lattice structures. Salts are the compounds which are formed by complete transfer of proton from one compound to another ${ }^{[18]}$. Salts and cocrystals can be differentiated based by a proton transfer from an acid to base. A complete transfer of proton takes place between acid-base pairs, whereas, no proton transfer occurs during cocrystal formation. Two components are bound to each other by noncovalent interactions such as hydrogen bonding, $\pi-\pi$ stacking, van der Waal forces. A prediction can be made by $\Delta \mathrm{pKa}$ value whether cocrystals are formed or not. It is generally accepted that a salt will be formed if the $\Delta \mathrm{p} K \mathrm{a}$ value is greater than 3 and $\Delta \mathrm{p} K$ a value less than 0 will lead to the formation of cocrystals. This parameter is not accurate to predict the formation of cocrystals in solids between the $\Delta \mathrm{pKa}$ values 0 and 3 but the possibility of salt formation will increase when the $\Delta \mathrm{pKa}$ increases ${ }^{[19,20]}$. Cocrystals and solvates can be differentiated based on their physical state of the components. The compounds which are liquid at room temperature are called as solvates whereas those compounds which are solid at room temperature are called as cocrystals. If the solvates contain water as a solvent in their crystal lattice then they are known as hydrates $^{[21]}$. Solvates/hydrates are commonly formed during the cocrystallization via solution or liquid assisted grinding ${ }^{[9]}$ and they can alter physicochemical properties of API's. Stability of solvates will be different from unsolvated forms because of presence of solvent in crystal lattice. Solvates/hydrates are quite unstable, because they lose solvent/water at high temperature and low humidity during storage and the physiochemical properties will be different for hydrated/dehydrated forms ${ }^{[22-24]}$. Dissolution rate of the drug was enhanced by the solvated forms of spironolactone ${ }^{[25]}$. Different polymorphic cocrystals and solvates of caffeine and anthranilic acid were prepared by using different solvents via liquid assisted grinding ${ }^{[26]}$.

\section{ADVANTAGES OF COCRYSTALS}

Physicochemical properties of drugs can be tailored by various approaches such as salt formation, micronization, solid dispersion, amorphous drugs and encapsulation. Among all these, the cocrystals should have the advantages that they will exist in stable crystalline form and no need of other excipients and additives in formulations ${ }^{[21]}$. The factors which play important role in affecting the physicochemical properties are the properties of APIs and coformers, the nature of molecular interaction between them and the employed synthetic procedures. The key advantage of formulating the cocrystals is that without altering the pharmacological properties, the APIs will benefit of their physicochemical properties enhancements because of the presence of coformer in crystal structure 
which is a property modifying component. The effect on the physicochemical properties of the API is dependent on the available coformer ${ }^{[16,24,27,28]}$. Another unique advantage of cocrystals over the more common salts is that cocrystals can be made for non-ionisable APIs as well as for those complex drugs which have sensitive functional groups that may not survive the harsh reaction conditions of strong acids or bases ${ }^{[12,28]}$. There are several other main advantages behind the formulating the cocrystals. Cocrystals have the potential to shorten the drug development timeline of APIs. Shortened development times equate to less cost, which is appealing to pharmaceutical companies. Solidstate synthesis techniques of cocrystals can be classified as green chemistry as they offer high yield, no solvent use and there are few by-products. Pharmaceutical cocrystals are structurally different to their bulk forms; it is possible to patent cocrystals of existing APIs as a new crystal form. Different formulations of pharmaceutical cocrystals are available in the market such as Viagra (Pfizer) to treat erectile dysfunction and pulmonary arterial hypertension, Entresto (Novartis) for treatment of chronic heart failure and some others under clinical developments ${ }^{[12,14,16,24,28]}$. Pharmaceutical cocrystals can enhance the physicochemical properties of drugs such as melting point, tabletability, solubility, stability, bioavailability, permeability and these properties are highlighted here with suitable examples.

\section{Melting point:}

Melting point is the physical property of solids, which is used to determine the purity of the product with sharp melts and narrow ranges ${ }^{[29]}$. High melting point demonstrates the thermodynamically stability of the new materials i.e. thermal stability of an API can be increased by selecting the coformer with higher melting point. Cocrystals with low melting points can also be beneficial when dealing with thermolabile drugs. The most commonly used techniques for determination of melting point and thermal analysis are differential scanning calorimetry (DSC) and thermal gravimetric analysis (TGA). Melting point of pharmaceutical cocrystals can be tailored by judicious selection of the coformers ${ }^{[24,30]}$. Melting point of about 50 cocrystals was analysed and the results showed that $51 \%$ cocrystals showed melting points between API and coformers, while $39 \%$ cocrystals have melting point lower than either API or coformer, $6 \%$ have high melting point than both API and coformer, and $4 \%$ had same about API or coformer ${ }^{[16]}$. Ten cocrystals of API AMG-517 was prepared and analysed that the melting points of cocrystals can be changed according to selected coformers. The results showed that the melting points of cocrystals and coformers are related to each other but melting point and solubility are not correlated to each other in a linear manner. For high melting point cocrystal, high melting point coformer should be used and vice-versa ${ }^{[31]}$. Similar types of results were also observed by many researchers i.e. melting point of cocrystals was directly related to the coformers' melting point whereas solubility of cocrystals was also not related to melting point directly. Less polar and hydrophobic coformer showed low aqueous solubility of cocrystals as compared to APIs itself ${ }^{[30,32]}$. Cocrystals of similar heterosynthons was prepared and the results showed different melting points of cocrystals because of different crystal packing ${ }^{[33]}$. Melting point contributes a major consideration during formulation of cocrystals. Cocrystals with high melting point are usually required but they have poor aqueous solubility whereas low melting point cocrystals have problems with processing, drying and stability, so further study within this area is required.

\section{Tabletability:}

Cocrystallization of drug and coformer can affect the crystal packing, tabletability and compaction, which are important parameters during preformulation study. Compaction behaviour of cocrystals of paracetamol with trimethylglycine and oxalic acid was found to be better than pure drug ${ }^{[34]}$. Tabletability of resveratrol was enhanced by formation of cocrystals with 4-aminobenzamide and isoniazid. Cocrystals showed higher tabletability than either pure drug or coformers $^{[35]}$. Mechanical properties of APIs could be altered by varying crystal packing by cocrystallization and cocrystals of vanillin isomers with same coformer showed higher tabletability than isomers and coformer ${ }^{[36]}$.

\section{Solubility:}

Solubility is an important parameter to investigate the formulations of poorly soluble drugs. Many approaches have been used to improve the solubility of drugs such as salt formation, solid dispersion, particle size reduction, and so on ${ }^{[23]}$, amongst which cocrystallization has been used by several researchers ${ }^{[37-41]}$. Solubility of antifungal drug ketoconazole was increased 53 and 100 times by synthesizing salts and cocrystals respectively as compared to ketoconazole. Thus, higher solubility of drug was obtained by cocrystals as compared to salt formation ${ }^{[37]}$. The solubility of 
apixaban cocrystals was increased about two times and cocrystals showed faster dissolution as compared to pure drug ${ }^{[38]}$. Six times enhancement of solubility was measured by formulating cocrystals of pterostilbene with piperazine whereas drug was precipitated rapidly in pterostilbene-glutaric acid cocrystals due to high solubility of glutaric acid ${ }^{[39]}$. Cocrystals of antitumor drug 6-mercaptopurine with nicotinamide showed two times higher dissolution as compared to pure drug ${ }^{[40]}$. A theoretical method based on $\mathrm{K}_{\mathrm{eu}}$ (the ratio of solution concentrations of cocrystal components at the eutectic point) was used to determine the cocrystals solubility in pure solvent and also a valuable tool for cocrystal selection and formulation without material and time requirement of traditional methods ${ }^{[42]}$. With the help of $\mathrm{K}_{\text {eu }}$, Serajuddin described the cocrystals solubility ratio and solution chemistry by using a set of more than 40 cocrystals and solvent combinations ${ }^{[43]}$. In one study, equations that describe cocrystal solubility in term of product solubility, cocrystal component ionization constants, and solution $\mathrm{pH}$ were derived for cocrystals with acidic, basic, amphoteric and zwitterionic components ${ }^{[12,44]}$.

\section{Stability:}

Stability study is extremely important during the development of new dosage formulation. During development of pharmaceutical cocrystals several stability studies should be performed such as relative humidity stress, chemical stability, thermal stability, solution stability and photostability study. In relative humidity stress, automated water sorption/desorption studies are performed to determine the effect of water on the formulation. Several researchers studied the behaviour of cocrystals under relative humidity stress conditions ${ }^{[41,45-47]}$. Cocrystals of glutaric acid and 2-[4-(4-chloro-2-fluorphenoxy)phenyl]pyrimidine4-carboxamide showed $0.08 \%$ moisture at high $95 \%$ $\mathrm{RH}$ and cocrystals were found stable at different conditions ${ }^{[41]}$. Indomethacin-saccharin cocrystals showed low water sorption during relative humidity studies and no dissociation or transformation occurred at experimental conditions ${ }^{[45]}$. Relative humidity stability behaviour of theophylline cocrystals with different coformers (oxalic acid, malonic acid, maleic acid and glutaric acid) was observed at different $\mathrm{RH}(0$, 43, 75 and 98\%) for different time intervals ( $1 \mathrm{~d}, 3 \mathrm{~d}, 1$ $\mathrm{w}$ and $7 \mathrm{w}$ ). The results showed the improvement in the physical property and stability especially by avoidance of hydrate formation ${ }^{[47]}$. During chemical stability study, any change or chemical degradation should be analysed in the formulation mainly at accelerated stability conditions. Very few reports were found about the chemical stability of cocrystals in the literature. Cocrystals of glutaric with an API did not show any degradation and showed good chemical stability at different conditions $\left(40^{\circ} / 75 \% \mathrm{RH}\right.$ and $\left.60^{\circ}\right)$ for $2 \mathrm{mo}^{[41]}$. Carbamazepine and saccharin cocrystals showed good chemical stability at different conditions $\left(5,40\right.$ and $60^{\circ}$ at ambient humidity and elevated RH stability at $25 \% 60 \%$ and $40^{\circ} / 75 \% \mathrm{RH}$ ) for $2 \mathrm{mo}^{[48]}$. High temperature stress can also be used to predict the physical and chemical stability based on accelerated stability conditions. Few researchers reported about thermal stability ${ }^{[49,50]}$. Paracetamol cocrystals with 4,4-bipyridine showed the better stability than other coformers upon heating by DSC ${ }^{[49]}$. Thermal stability of cocrystals (L-883555, a phosphodiesterase IV inhibitor) with tartaric acid was studied in different stoichiometries ranging from $0.3: 1.0$ to $0.9: 1.0$. Cocrystals with stoichiometries with 0.5:1.0 was found to be most stable because acid content can occupy the channels in the crystals and establish multiple binding modes ${ }^{[50]}$. Solution stability is an important parameter for development of cocrystals to determine the stability in the solution. Solution stability studies give better understanding about the cocrystal behaviour in the release media ${ }^{[16]}$. Behaviour of carbamazepine cocrystals was studied in water for 20-48 $\mathrm{h}$ and found that the cocrystals with high water soluble coformers converted into dihydrates, while cocrystals with low water soluble coformers remained as such in the solution ${ }^{[51]}$. Caffeine/oxalic acid cocrystals showed better stability than others at all $\mathrm{RH}$ up to $98 \%$ for $7 \mathrm{w}$ and no significant change was observed in their physical form when materials were slurried in water at ambient temperature for two days ${ }^{[22]}$. The stability of carbamazepine and saccharine cocrystals was determined by slurring the materials with equal parts in water and after $24 \mathrm{~h}$, powder X-ray diffraction (PXRD) analysis showed that only cocrystals were present in the solution, no other form was detected ${ }^{[48]}$. Photostability study is performed to study the effect of light on light sensitive drugs. Many drugs are unstable in light and so photostability study is necessary for these types of drugs. Very few reports were found about the chemical stability of cocrystals in the literature. Nitrofurantoin cocrystals with different coformers showed a higher photostability as compared to pure drug and physical mixture. All the cocrystals showed little degradation ( $<3 \%)$ except one after $168 \mathrm{~h}$ and thus, photo degradation of light sensitive drug can be prevented by cocrystallization ${ }^{[53]}$. 


\section{Bioavailability:}

Bioavailability is defined as the rate and extent of pure drug that reaches into systemic circulation ${ }^{[54]}$. Low oral bioavailability of APIs is a major challenge during the development of new formulations. Crystal engineering is mainly used to design and synthesize the pharmaceutical cocrystals with enhanced aqueous solubility and oral bioavailability. Pharmacokinetics study of apixaban-oxalic acid cocrystals in beagle dogs showed that oral bioavailability was increased by 2.7 times as compared to pure drug ${ }^{[38]}$. Oral bioavailability of baicalein was increased by formation of cocrystals with nicotinamide and showed that cocrystals had 2.49 times higher peak plasma concentration $\left(\mathrm{C}_{\max }\right)$ and 2.80 times higher area under the curve (AUC) as compared to pure drug in rats ${ }^{[55]}$. Meloxicam cocrystals with aspirin exhibited better oral bioavailability as compared to pure drug and showed 12 times faster onset of action than pure drug in rats ${ }^{[56]}$. Cocrystals of 6-mercaptopurine (a BCS Class-II drug) showed higher oral bioavailability i.e. $168.7 \%$ than pure drug in rats ${ }^{[40]}$.

\section{Permeability:}

Drug absorption and distribution of drugs mainly depends upon the permeability of drugs across the biological membrane. Permeability of drugs mainly depends upon the n-octanol/water partition coefficient by using $\log \mathrm{P}$ and $(\mathrm{C} \log \mathrm{P})$ for unchanged form of drug ${ }^{[28]}$. Permeability of a BCS class-III drug, 5-fluorouracil, was enhanced by cocrystallization with different coformers such as 3-hyroxybenzoic acid, 4-aminobenzoic acid and cinnamic acid ${ }^{[57]}$. Permeability study of hydrochlorothiazide and cocrystals with different coformers was studied by using Franz diffusion cells. The amount of drug flux in all cocrystals was higher as compared to pure drug except for succinamide cocrystals. Cocrystals permeability was improved due to formation of heterosynthon between drug and coformer ${ }^{[58]}$.

\section{SELECTION OF COFORMERS AND SCREENING OF COCRYSTALS}

A pharmaceutical co-crystal is a single crystalline solid that incorporates two neutral molecules, one being an API and the other a co-crystal former. Co-crystal former may be an excipient or another drug ${ }^{[59]}$. The USFDA had maintained a list of substances which is numbering in thousands and can be used as potential coformer for pharmaceutical cocrystals ${ }^{[60]}$. The non API component used as coformer should be non-toxic with no adverse side effects. Ideally, the cocrystal former should be included on the US FDA "Everything added to food in the United States" (EAFUS) list, which comprises over 3000 substances that are suitable as food additives, or approved as Generally Regarded as Safe (GRAS) ${ }^{[61]}$. Coformer selection for an API is the most important aspect for designing and screening of cocrystals. Selection of coformers is mainly done by different methods such as knowledge based methods and experimental methods. Hit and trial approach is mainly used with all types of coformers for an API and to confirm the structure of cocrystal, these are characterized by suitable techniques. This technique is expensive and time consuming. For selection of suitable coformers and screening of cocrystals, researchers have used some different knowledge based approaches which include the followings: hydrogen-bonding propensity, synthonic engineering, supramolecular compatibility by Cambridge Structure Database (CSD), pKa based models, Fabian's method, Lattice energy calculation, the conductor-like screening model for real solvents (COSMO-RS), Hansen solubility parameter, virtual cocrystal screening (based upon molecular electrostatic potential surfaces-MEPS), thermal analysis, measuring saturation temperature, Kofler contact method and matching $^{[10,62]}$.

\section{Hydrogen bonding propensity:}

In cocrystals, API and coformers interact with each other by non-covalent bonding such as hydrogen bonding and van der Waal forces. Among all of these, hydrogen bonding between API and coformer plays an important role in the formation of cocrystals $\mathrm{s}^{[7,8]}$. Etter described a graph-set notation system which has been mostly used as motif for labelling of hydrogen bonding ${ }^{[6]}$ and suggested 3 rules for preferable hydrogen bond formation: every hydrogen molecule, which is acidic in nature will be present in bond formation, all hydrogen bond acceptors will be used when there are available hydrogen bond acceptors, and hydrogen bond will be formed when there will be best hydrogen bond donors and hydrogen bond acceptors ${ }^{[7]}$. The quantitative measurement of hydrogen bond formation between donor and acceptor functional groups present in indomethacin and isonicotinamide was analysed by assigning a value between 0 to 1 and higher value indicates the formation of hydrogen bond ${ }^{[63]}$.

\section{Synthonic engineering:}

Desiraju described the "synthon approach" for the selection of coformers which formed a supermolecule 
by using specific molecular fragments within the cocrystal to establish "supramolecular synthons" According to this approach, the functional groups present in API and coformer will play a major role in the formation of cocrystals and coformer with suitable functional group will be used for a particular APIs. Synthons are present in the supermolecules as basic structural units which are associated with noncovalent bonding. Supramolecular synthon approaches are of two types: supramolecular homosynthons and supramolecular heterosynthons. Supramolecular homosynthons are composed by same functional groups present in API and coformer such as the amide-amide homosynthons, carboxylic acid-acid homosynthons whereas supramolecular heterosynthons are formed by different functional groups such as the carboxylic acid-amide heterosynthons, the acid-pyridine heterosynthons ${ }^{[24]}$. Supramolecular heterosynthons are generally more favoured than homosynthons, e.g., the acid-amide and the acid-pyridine heterosynthons are commonly used as compared to carboxylic acid and amide homodimers $^{[22]}$.

\section{CSD:}

CSD is a validated tool to facilitate the statistical analysis of packing motifs and thereby provide information about common functional groups. CSD is used to provide the information about molecular association of drug and coformers based on functional group that engage into supramolecular synthons. A library of suitable coformers can be prepared by CSD for an API. This is a computer based approach used to find appropriate cocrystal forming pairs, and reduces the research time and experimental cost ${ }^{[12,22,24]}$.

\section{pKa rule:}

Cocrystals or salts formation can be predicted by proton transfer between acid and base. The formation of salts or cocrystals can be predicted by determining the $\Delta \mathrm{p} K \mathrm{a}=[\mathrm{pKa}$ (base) $-\mathrm{pKa}$ (acid)]. It is generally accepted that proton transfer will occur from acid to base if the difference in the $\mathrm{pKa}$ values is greater than 2 or 3 . A smaller $\Delta \mathrm{pKa}$ value (less than 0 ) indicates the formation of cocrystals whereas higher value (more than 2 or 3 ) indicates the formation of salts ${ }^{[19,20]}$. $\triangle \mathrm{p} K \mathrm{a}$ rule was validated and quantified by studying 6465 cocrystals from CSD and explained a linear relationship between $\Delta \mathrm{pKa}$ value and possibility of proton transfer between acid-base pair. It was analysed that a non-ionized complex should be formed when the value of $\Delta \mathrm{pKa}<-1$; an ionized complex is formed when the value of $\Delta \mathrm{pKa}<4$ and the possibility of formation of ionized complex increase by $17 \%$ by increase in $\Delta \mathrm{pKa}$ by one unit from $10 \%$ at $\Delta \mathrm{pKa}=-1$ to $95 \%$ at $\Delta \mathrm{pKa}=4$. By determining the $\Delta \mathrm{pKa}$ value, the possibility of formation of cocrystals and salts can be determined. This is a simple and less time-consuming method for the preparation of cocrystals ${ }^{[64]}$.

\section{Fabian's method:}

Different sets of reliable cocrystal forming structures were extracted from the CSD and the molecular descriptors (single atom, bond and group counts, hydrogen bond donor and acceptor counts, size and shape, surface area and molecular electrostatic) were calculated for each molecule. On the basis of calculated molecular properties, the database described pairs of molecules that were able to form cocrystals. The strongest descriptor correlation was related to the shape and polarity of cocrystal formers ${ }^{[65]}$.

\section{COSMO-RS:}

For screening of suitable coformers for an API, COSMO-therm software based on COSMO-RS fluidphase thermodynamic approach was used to describe the miscibility of coformers in super cooled liquid (melt) phase. The excess enthalpy, $\mathrm{H}_{\mathrm{ex}}$ (a major factor for H-bonding interactions) between API and coformer mixture as compared to pure components reflects the tendency of those two compounds to cocrystallize. This was demonstrated that COSMO-RS theory allowed reasonable ranking of coformers for an API and the experiments should be focused on those coformers which an increased probability of cocrystallization, leading to the largest improvement of the API solubility. In a similar way as potential coformers were identified for cocrystallization, solvents with highest value of $\mathrm{H}_{\text {ex }}$ with an API, were selected which had the least probability to form solid solvates. Different coformers for itraconazole and solvents for Axitinib (tyrosine kinase inhibitor) were selected by this method to avoid formation of hydrates and solvents ${ }^{[66]}$.

\section{Hansen solubility parameter:}

Hansen solubility parameter is another important approach used to measure the miscibility of drug and coformers used for cocrystal systems. The miscibility of the components in the solid state could predict the cocrystal formation. The synthesis of cocrystals success rate was improved by using the components which have similar miscibility ${ }^{[67]}$. It was demonstrated that the two components should be miscible if total HSPs difference 
was $\quad<7 \mathrm{MP}_{\mathrm{a}}^{0.5}$, otherwise immiscible ${ }^{[68]}$. Another method estimates the miscibility of two components if the difference is $\leq 5 \mathrm{MP}^{0.5}$ between two substances which are supposed to be cocrystal formation ${ }^{[69,70]}$.

\section{Virtual cocrystal screening:}

Musumeci et al. predicted that all the possible intermolecular interaction sites present on the surface of molecules were responsible for the formation of cocrystals $^{[71]}$. The strength of hydrogen bond depends upon the H-bond donors and H-bond acceptors; best $\mathrm{H}$-bond donor and best H-bond form the strongest $\mathrm{H}$-bond, and next best $\mathrm{H}$-bond acceptor interacted with the next H-bond donor, and so on, until all sites are satisfied. Calculated gas phase MEPS approach has been used for screening of cocrystals. This approach assumed that the energy difference, $\Delta \mathrm{E}$, between the two pure solids and cocrystals in different stoichiometries give an idea about the cocrystal formation. The results showed that the probability of cocrystal formation was $50 \%$ more, when $\Delta \mathrm{E}$ difference of cocrystals and two pure solids should be more than $11 \mathrm{~kJ} / \mathrm{mol}$. This approach was validated by using about 1000 compounds from literature for APIs (caffeine and carbamazepine) and $\Delta \mathrm{E}$ parameter was found to be favourable and fast screening tool ${ }^{[71]}$.

\section{Cocktail cocrystal method:}

A new "cocrystal cocktail method" was developed for screening of cocrystals formation in which four coformers were ground simultaneously with API in ball mill, this method reduced the workload by $50 \%$ and hence it was convenient and less time consuming as compared to conventional single time-consuming methods. The chemical moieties present in coformers and drug interacted with each other and synthons were formed between drug and coformers i.e. homosynthons or heterosynthons ${ }^{[72]}$. Cocrystals of itraconazole was prepared with succinic acid and serine by using this method and the results showed the highest in vitro solubility and dissolution rate as compared to all other formulations ${ }^{[70]}$.

\section{Thermal analysis:}

A rapid thermal method i.e. DSC has been used for the screening of the cocrystals ${ }^{[73]}$. The possibility of formation of cocrystal can be determined by heating the physical mixture (1:1) in DSC. A hypothesis was proposed that three endotherms and two exotherms represented the cocrystals formation with stoichiometric diversity, two endotherms and one exotherm represented one cocrystal formation with certain molar ratio and one endotherm represented no cocrystal formation ${ }^{[74]}$. The physical mixture of 20 reported drug-coformer systems were analysed by DSC and found an exothermic peak was always associated with formation of the formation of cocrystals ${ }^{[75]}$. DSC has been used for cocrystal screening and compatibility study of API and coformer. This is very rapid screening method for cocrystal formation and a small amount of sample is used to analysis. No solvent required in this method, so it is also known as "green technique". But, this technique is not suitable for those compounds which are thermally unstable and volatile in nature. During scanning, some physical transformations take place, which make this complicate to understand properly ${ }^{[73]}$.

Thermal screening of cocrystals by DSC gave some ambiguous results. So, some researchers used DSC with other techniques for the screening of the cocrystals $^{[76]}$. Hot stage microscopy was also used for identification of cocrystals and found to increase the overall screening efficiency ${ }^{[77]}$. This method offers identification of number of phases present in the system by direct visualization when two components were heated. High melting point component melted and recrystallized before another molten component came in contact with it, and zone of mixing was created. In this method, thermodynamic landscape was elucidated with the help of binary phase diagram and efficiency of cocrystal screening was increased. Nicotinamide was selected as coformer and hot stage microscopy was used for screening of cocrystals' formation with different APIs. Hot stage microscopy is also known as Kofler contact method ${ }^{[77,78]}$.

A new method was developed for the screening of the cocrystals by measuring the saturation temperature of cocrystals and their components ${ }^{[15]}$. Saturation solubility of drug and coformer is determined separately at particular temperature known as reference temperature and saturation temperature of the solution is determined by heating with $0.3^{\circ} / \mathrm{min}$. The increase in saturation temperature more than $10^{\circ}$ from reference temperature is the possibility of cocrystal formation. Manin et al., evaluated the relative efficiency of DSC, hot stage microscopy and saturation temperature method. Among different methods for cocrystal screening and saturation temperature method was found to be most effective than DSC and HSM. However, it requires the use of solvent and is most time-consuming method ${ }^{[79]}$. 


\section{Synthon matching:}

Synthon matching is the computational theory used to investigate the intermolecular interactions in the crystal structure and is an important tool for the cocrystal screening. The major limitation of this approach is that in vivo properties of cocrystals cannot be determined exactly. This synthon approach is used to estimate the possibility of hydrogen bond formation between API and coformer ${ }^{[24]}$. Over the past few years, various methods have been evolved to determine the intermolecular interactions in crystal structures qualitatively and quantitatively, such as the conformational similarity index for proteins, graphset analysis for hydrogen bonds, Voronoi-Dirichlet polyhedral for crystal packing, continuous symmetry measures, and the Hirshfeld surface by using computer programs such as ESCET, COMPACK, TOPOS, Xpac, Crystal Explorer, and dSNAP, respectively ${ }^{[80]}$.

\section{DIFFERNT METHODS OF COCRYSTALS FORMATION}

Till date, different methods have been reported for the preparation of cocrystals by the researchers. Few traditional methods based on the solution and grinding were reported for the synthesis of cocrystals ${ }^{[81]}$. A suitable type of solvent is used in solution method for the preparation of cocrystals. Different types of solution methods such as solvent evaporation ${ }^{[76]}$, solution crystallization technique ${ }^{[41]}$, antisolvent addition $^{[82]}$, slurry conversion method ${ }^{[83]}$ and reaction crystallization method ${ }^{[84]}$ are discussed with suitable examples. Grinding methods are of two types: neat grinding and solvent drop grinding ${ }^{[85,86]}$. Some newly emerging methods used for the formation of cocrystals are ultrasound assisted solution method ${ }^{[87,88]}$, supercritical fluid atomization technique ${ }^{[46,89]}$, spray drying technique ${ }^{[00,91]}$, hot melt extrusion technique ${ }^{[92,93]}$.

\section{Solution-based methods:}

In solvent evaporation method, for both API and coformer are dissolved in a suitable solvent and the solution is allowed to evaporate the solvent slowly. During dissolution, the functional groups in the drug and conformer interact with each other and form hydrogen bonds ${ }^{[89]}$. This is most commonly used method for the preparation of cocrystals by researchers ${ }^{[79,94]}$.

In solution crystallization technique, drug and coformers are dissolved in boiling solvent with stirring and the boiling of the solution would be continued until the volume of the solution become small.
Cocrystallization takes place rapidly when the boiling solution is allowed to cool about $15 \mathrm{~min}$. Cocrystals are separated by filtration and kept in oven or air for drying ${ }^{[41,95]}$.

Slurry crystallization is the process in which slurry is prepared by addition of different solvents in the mixture of API and suitable coformers. The solvent is decanted and the solid material is dried and characterized by different methods for evaluation. This method is selected for the preparation of cocrystals when the drug and coformer should be stable in the solvent ${ }^{[29,83,96,97]}$.

In antisolvent addition method, the coformers are dissolved in different solvents such as organic solvents and API is dispersed in the coformer solution by using dispersion homogenizer. This solution is then added to distilled water or suitable solution to precipitate the coformer on the drug ${ }^{[82,98,99]}$.

Reaction crystallization method is used for rapid preparation of cocrystals at microscopic and macroscopic scale at ambient temperature in which nucleation and cocrystallization is based upon the cocrystal components and their solubility. The saturated solution of the lesser soluble component (drug) is made in methanol and filtered, and then the more soluble component (coformer) is added in an amount just under its solubility limit. The goal is not to have any excess drug or coformer in the starting solutions that could be confused as a cocrystal. Furthermore, by not exceeding the solubility limits of the components, the cocrystals that precipitate out of solution are pure. Solution concentrations are monitored by HPLC throughout the crystallization process to evaluate whether the solid observed appeared to be a complex of the reactants (cocrystals). The solid precipitates are also collected and analysed by HPLC to determine the stoichiometry of the complex. If the solid appeared to be a cocrystal based on the HPLC results, it is further characterized by DSC, TGA, and PXRD ${ }^{[84,100,101]}$.

\section{Grinding method:}

Grinding methods have been widely used for the for the cocrystal formation over the past few years and found to be superior than other methods (solution or melt ${ }^{[85,86]}$. Grinding techniques are of two types: neat or dry grinding and wet grinding. In dry grinding, drug and coformer are mixed together in a stoichiometric ratio and ground them by using either mortar and pestle or ball mill[102]. Wet Grinding was performed in a similar manner that of neat grinding by addition of some drops of solvent in the mixture ${ }^{[103,104]}$. 


\section{Ultrasound assisted solution cocrystallization:}

Sonochemical method has been developed for the preparation of cocrystals of very small size i.e. for preparation of nanocrystals ${ }^{[87]}$. In this method, API and cocrystal former are dissolved together in a solvent and the solution is kept in a sonoreactor to form the solution turbid. Cold water is supplied during the sonication to maintain the constant temperature of sonicator and prevent fragmentation. The solution is kept overnight for drying. Pure cocrystals were obtained by this method and the purity of cocrystals can be assessed by using X-ray diffraction study ${ }^{[88]}$.

\section{Supercritical fluid atomization technique:}

In supercritical atomization technique, the drug and coformers are mixed with each other by using high pressurized supercritical fluid i.e. $\mathrm{CO}_{2}$. Cocrystals are prepared by atomizing this solution with the help of atomizer. In supercritical antisolvent (SAS) method, the cocrystals are prepared from solution by the antisolvent effect of supercritical fluid ${ }^{[46,89,105]}$.

\section{Spray drying technique:}

In spray drying process, cocrystals are prepared by spraying the solution or suspension of drug and coformer with hot air stream to evaporate the solvent. This is the most preferred technology because this is a fast, continuous, and one-step process. Thus, spray drying process will offer a unique environment for the preparation and scale-up of cocrystals ${ }^{[90,91]}$.

\section{Hot melt extrusion technique:}

In hot melt extrusion technique, the cocrystals are prepared by heating the drug and coformers with intense mixing which improved the surface contacts without use of solvent. The limitations of this method include both coformer and API should be miscible in molten form and not used for thermolabile drugs ${ }^{[29,92,93]}$.

\section{EVALUATION OF COCRYSTALS}

FTIR spectroscopy is used to predict the intermolecular interactions and compatibility study between drug and coformers. This technique is widely used to predict the chemical conformation of compounds. Aakeroy et al. used FTIR to distinguish the cocrystals from salts by evaluating the carboxylic acid involvement in the hydrogen bond formation ${ }^{[106]}$. Pure drug, coformer, physical mixture and cocrystals are analysed by FTIR in the range of $400-4000 \mathrm{~cm}^{-1}$. FTIR study is also used along with other techniques such as DSC or XRD for the screening of the cocrystals ${ }^{[12,76,107]}$.
DSC has been used for screening of cocrystal formation. Screening of cocrystals formation can be determined by the presence of exothermic peak followed by endothermic peak in DSC spectra. The presence of these peaks in the physical mixture of components indicates the possibility of formation of cocrystals. Pure drug, coformer, physical mixture and cocrystals were weighed out (1.5-2.5 mg) in aluminium pans and analysed with heating rates of $5-30^{\circ}$ using similar empty pan as a reference. The nitrogen gas with flow rate 50 $\mathrm{ml} / \mathrm{min}$ maintained the inert atmosphere. Melting point, glass transition temperature, polymorphic nature, heat of fusion, endothermic or exothermic behaviour can be determined by using DSC ${ }^{[73,75,98]}$.

Physical and chemical properties of solids are determined by using thermal analysis as a function of increasing temperature (with constant heating rate) or as a function of time (with constant temperature and/or constant mass loss). TGA is a suitable method for determination of hydrates/solvates forms of cocrystals or presence of volatile components as well as decomposition or sublimation temperature. Thermal stability, compatibility and purity of cocrystals can be predicted by TGA analysis. The weight loss of sample mass during the TGA analysis is the indication of loss of volatile component or decomposition of cocrystal ${ }^{[61,108]}$.

Terahertz time-domain-spectroscopy (THz-TDS) is an alternative tool to PXRD for the characterization of cocrystals. Chiral and racemic molecular and supramolecular structures can be distinguished by terahertz spectroscopy ${ }^{[12]}$. Terahertz spectroscopy was used to distinguish the identical molecular structure cocrystals of theophylline with different coformers (such as malic acid and tartaric acid) which were present in chiral and racemic forms ${ }^{[109]}$.

Solid-state NMR (SSNMR) is used to characterize solid phases that cannot be studied by SXRD ${ }^{[12]}$. SSNMR was used to investigate the nature of complex by determining degree of proton transfer. Thus, SSNMR is an important tool for the identification of cocrystal or salt. SSNMR can also be used to evaluate the cocrystal structure by estimating hydrogen bonding and local conformation changes by couplings ${ }^{[110,111]}$.

PXRD is commonly used for screening and determination of cocrystalstructure ${ }^{[89]}$. The PXRD patterns obtained from diffractometer were compared to each other for analysing the structure of cocrystals. The different PXRD pattern of cocrystals from their components is the indication of cocrystal 
formation $^{[103,112]}$. Crystal structure of solids at atomic level cocrystals is determined by using single crystal $\mathrm{X}$-ray diffraction (SXRD). The major problem related to this technique is that generally single cocrystal cannot be produced which is suitable for SXRD analysis ${ }^{[12]}$. Scanning electron microscope is the instrument used to determine the particle size and morphological analysis of cocrystals. A high energy electron beams scan the atoms that provide the information about the sample surface's topography ${ }^{[12,45,46]}$.

Dissolution study is used to determine the amount of drug release with time in dissolution medium and predict the in vivo performance of the formulation. Dissolution studies for the cocrystals can be performed with the help of the dissolution apparatus. The dissolution studies for the cocrystals can be done within the suitable dissolution medium described in drug protocol of referred pharmacopoeia. The drug samples can be collected in the suitable quantity at predetermined time interval and can be examined with the help of suitable means like HPLC or UV ${ }^{[113,114]}$.

Solubility study can be assessed by Higuchi and Connors method for solubility determination. The solubility of pure drug, physical mixture and cocrystals can be determined in water or suitable medium given in the referred pharmacopoeia. Drug sample and medium should be added in a conical flask, and should be shaken for $24 \mathrm{~h}$ at room temperature on rotary flask shaker. The entire samples should be protected from light by wrapping the flask by aluminium foil if the drug is sensitive to light. After $24 \mathrm{~h}$ samples are filtered through Whatman filter paper and aliquots are suitably diluted and assayed by HPLC or UV at suitable wavelength $^{[115,116]}$.

Stability study provides the information about shelf life of drug products under different storage conditions. Drugs products should be kept in glass vials under variable environmental factors (such as humidity, temperature, light) for different intervals of time. After that, the samples are analysed for thermal study, drug release study, XRD study and FTIR study and compared with the results obtained before stability study ${ }^{[98]}$.

\section{APPLICATIONS}

Cocrystallization has an advantage to optimize the physicochemical properties of drugs without altering the molecular structure of drugs. The chew over whether cocrystals or salts will have the desired properties depends upon the API and specific project.
Sometimes salts have better physicochemical properties such as salts have higher intrinsic solubility in water than cocrystals. Cocrystals with negative $\Delta \mathrm{pKa}$ value will give non-ionized drug when dissolved whereas salt will give ionized API, which is more soluble in water. Whenever dissolution rate of drug should be important rather than equilibrium solubility, cocrystals can be better than salt form of drug. Cocrystallization is an alternative way to enhance the solubility and bioavailability of poorly water soluble drugs, especially for those compounds which are neutral or weakly ionized in nature ${ }^{[12,20,37]}$. Further, cocrystallization also offers possibility of altering improving the melting point, tabletability, solubility, stability, bioavailability and permeability. as discussed in previous sections.

\section{FUTURE PERSPECTIVES AND CHALLENGES}

Cocrystallization is a viable approach for the enhancement of physicochemical properties of drugs while preserving the pharmacological property of the API. Selection of suitable coformers for an API is one of the major challenges in the formation of pharmaceutical cocrystals. Various approaches have been used for coformer selection and screening of cocrystals, however each approach has its own limitations. Mainly, those compounds should be used as coformers which are listed as GRAS by USFDA and EAFUS database but GRAS status does not guarantee its use as cocrystal forming agents. Stability in presence of excipients is also an issue in the development of cocrystals and currently this is an unexplored area. Some other issues are related to the scale up of the production of high purity cocrystals, which makes cocrystals unattractive option for industry. In 2011, US-FDA released guidelines for the pharmaceutical industry, pertaining to patenting of cocrystals. FDA classified cocrystals as an "API excipients" molecular complex, a drug product intermediate and not a new API. On the other hand, EMA state that cocrystals should undergo similar principles of documentation as salt. Thus, although the regulatory perspectives of USFDA and EMA are different, it does demonstrate the growing interest in the use of pharmaceutical cocrystals as potential marketable drugs. Development, screening and evaluation of new cocrystals of drugs require a lot of time and energy; however, various researchers have used some knowledge based approaches for selection of coformers, designing and screening of cocrystals, as highlighted in earlier sections ${ }^{[10,24,116]}$. Research into 
cocrystals continues to grow and as more drug products that are based on cocrystals research hit the market, it can only be expected for pharmaceutical cocrystals to gain a stronger grip in drug development.

In the development of oral formulations, poor aqueous solubility and low bioavailability of drugs are major challenges. Amongst the various techniques for improvement of these challenges, cocrystals approach offer unique advantages of retaining the pharmacological properties of the drug, while benefitting from the physicochemical properties of the coformer. The major advantage of cocrystals over salts is that cocrystals can be used for those drugs which are non-ionisable or weakly ionisable in nature. Thus, cocrystals offer potential of improvement of melting point, tabletability, solubility, stability, bioavailability and permeability, besides simple formulation approaches. Cocrystals approach is not yet widely explored, and a combination of knowledge based and experimental methods for coformer selection offer a new era in cocrystal formation. Industrial interest in pharmaceutical cocrystals is growing due to the enhanced pharmaceutical benefits they exhibit and the decreased drug development time in cocrystals approach.

\section{Conflict of interest:}

The authors report no declarations of interest.

\section{Financial support and sponsorship:}

Nil.

\section{REFERENCES}

1. Babu NJ and Nangia A. Solubility advantage of amorphous drugs and pharmaceutical cocrystals. Cryst Growth Des 2011;11:2662-79.

2. Fong SYK, Ibisogly A, Bauer-Brandl A. Solubility enhancement of BCS class-II drug by solid phospholipid dispersions: Spray drying versus freeze-drying. Int J Pharm 2015;496:382-91.

3. Yuvaraja K, Khanam J. Enhancement of carvedilol solubility by solid dispersion technique using cyclodextrins, water soluble polymers and hydroxyl acid. J Pharm Biomed Anal 2014;96:10-20.

4. Hisada N, Takano R, Takata N, Shiraki K, Ueto T, Tanida S, et al. Characterizing the dissolution profile of supersaturable salts, cocrystals and solvates to enhance in vivo oral absorption. Eur J Pharm Biopharm 2016;103:192-9.

5. Savjani KT, Gajjar AK, Savjani JK. Drug solubility: Importance and enhancement techniques. ISRN Pharm 2012;2012:195727.

6. Etter MC. Encoding and decoding hydrogen-bond patterns of organic compounds. Acc Chem Res 1990;23:120-6.
7. Etter MC. Hydrogen bonds as design elements in organic chemistry. J Phys Chem 1991:95;4601-10.

8. Desiraju GR. Supramolecular synthons in crystal engineering - a new organic synthesis. Angew Chem Int Ed Engl 34:2311-27.

9. Almarsson O, Zaworotko MJ. Crystal engineering of the composition of pharmaceutical phase. Do pharmaceutical cocrystals represent a new path to improved medicines? Chem Commun 2004:1889-96.

10. Duggirala NK, Perry ML, Almarsson O, Zaworotko MJ. Pharmaceutical cocrystals: along the path to improved medicines. Chem Commun 2016;52:640-55.

11. Braga D, Grepioni F, Maini L, Prosperi S, Gobetto R, Chierotti MR. From unexpected reactions to a new family of ionic cocrystals: the case of barbituric acid with alkali bromides and caesium iodide. Chem Commun 2010:46;7715-7.

12. Qiao N, Li M, Schlindwein W, Malek N, Davies A, Trappitt G. Pharmaceutical cocrystals: An overview. Int J Pharm 2011;419:1-11.

13. Aakeroy CB, Salmon DJ. Building cocrystals with molecular sense and supramolecular sensibility. Cryst Eng Comm 2005;7(72):439-48.

14. Shan N, Zaworotko MJ. The role of cocrystals in pharmaceutical sciences. Drug Discov Today 2008;13:440-46.

15. Horst JHT, Deji MA, Cains PW. Discovering new cocrystals. Cryst Growth Des 2009;9(3):1531-7.

16. Schultheiss N, Newman A. Pharmaceutical cocrystals and their physicochemical properties. Cryst Growth Des 2009;9:295067.

17. Aitipamula S, Banerjee R, Bansal AK, Biradha K, Cheney ML, Choudhury AR, et al. Polymorphs, salts and cocrystals: What's in a name? Cryst Growth Des 2012:12:2147-52.

18. http://www.fda.gov/downloads/drugs/ guidancecomplianceregulatoryinformation/guidances/ ucm281764.pdf.

19. Bhogala BR, Basavoju S, Nangia A. Tape and layer structures in cocrystals of some di- and tricarboxylic acids with 4,4-bipyridines and isonicotinamide. From binary to ternary cocrystals. CrystEngComm 2005:7;551-62.

20. Childs SL, Stahly GP, Park A. The salt-cocrystals continuum: The influence of crystal structure on ionization state. Mol Pharm 2007:4;323-38.

21. Morissette SL, Almarsson O, Peterson ML, Remenar JF, Read MJ, Lemmo AV, et al. High-throughput crystallization: polymorphs, salts, cocrystals and solvates of pharmaceutical solids. Adv Drug Deliv Rev 2004:56;275-300.

22. Vishweshwar P, McMahon JA, Bis JA, Zaworotko MJ. Pharmaceutical cocrystals. J Pharm Sci 2006;95:499-516.

23. Blagden N, Matas M, Gavan PT, York P. Crystal engineering of active pharmaceutical ingredients to improve solubility and dissolution rates. Adv Drug Deliv Rev 2007:59;617-30.

24. Ross SA, Lamprou DA, Douroumis D. Engineering and manufacturing of pharmaceuticals cocrystals: a review of solvent free manufacturing technologies. Chem Commun 2016;52:8772-86.

25. Salole EG, Al-Sarraj FA. Spiranolactone crystal forms. Drug Dev Ind Pharm 1985:11;855-64.

26. Madusanka N, Eddleston M, Arhangelskis M, Jones W. Polymorphs, hydrates and solvates of a co-crystal of caffeine with anthranilic acid. Acta Crystallogr B Struct Sci Cryst Eng Mater 2014:70;72-80. 
27. Sekhon BS. Pharmaceutical cocrystals - An update. Chem Inform 2013;44:62.

28. Bolla G, Nangia A. Pharmaceutical cocrystals: walking the talk. Chem Commun 2016:52;8342-60.

29. Abourahma H, Cocuzza DS, Melendez J, Urban JM. Pyrazinamide cocrystals and the search for polymorphs. CrystEngComm 2011;13:1-22.

30. Batisai E, Ayamine A, Kilinkissa OEY, Bathori N. Melting point-solubility-structure correlations in multicomponent crystal containing fumaric or adipic acid. CrystEngComm 2014:16;9992-8.

31. Stanton MK, Bak A. Physicochemical properties of pharmaceutical cocrystals: A case study of ten AMG 517 cocrystals. Cryst Growth Des 2008:8;3856-62.

32. Aakeroy CB, Forbes S, Desper J. Using cocrystals to systematically modulate aqueous solubility and melting behaviour of an anticancer drug. J Am Chem Soc 2009:131;17048-9.

33. Fleischman SG, Kuduva SS, McMahon JA, Moulton B, Walsh $\mathrm{B}$, Rodriguez-Hornedo $\mathrm{RD}$, et al. Crystal engineering of the composition of pharmaceutical phases: multiple-component crystalline solids involving carbamazepine. Cryst Growth Des 2003:3;909-19.

34. Maeno Y, Fukami T, Kawahata M, Yamaguchi K, Tagami T, Ozeki $\mathrm{T}$, et al. Novel pharmaceutical cocrystal consisting of paracetamol and trimethylglycine, a new promising cocrystal former. Int J Pharm 2014:473;179-86.

35. Zhou Z, Li W, Sun WJ, Lu T, Tong HHY, Sun CC, et al. Resveratrol cocrystals with enhanced solubility and tabletability. Int J Pharm 2016:509;391-9.

36. Krishna GR, Shi L, Bag PP, Sun CC, Reddy CM. Correlation among crystal structure, mechanical behaviour and tabletability in cocrystals of vanillin isomers. Cryst Growth Des 2015:15;1827-32.

37. Martin FA, Pop MM, Borodi G, Filip X, Kacso I. Ketoconazole salts and cocrystals with enhanced aqueous solubility. Cryst Growth Des 2013:13;4295-304.

38. Chen Y, Li L, Yao J, Ma YY, Chen JM, Lu TB. Improving the solubility and bioavailability of Apixaban via ApixabanOxalic acid cocrystal. Cryst Growth Des 2016:16;2923-30.

39. Bethune SJ, Schultheiss N, Henck JO. Improving the poor aqueous solubility of nutraceutical compound Pterostilbene through cocrystal formation. Cryst Growth Des 2011:11;281723.

40. Wang JR, Yu X, Zhou C, Lin Y, Chen C, Pan G, et al. Improving the dissolution and bioavailability of 6-mercaptopurine via cocrystallization with isonicotinamide. Bioorg Med Chem Lett 2015:25;1036-9.

41. McNamara DP, Childs SL, Giordano J, Iarriccio A, Cassidy $\mathrm{J}$, Shet MS, et al. Use of a glutaric acid cocrystal to improve oral bioavailability of a low solubility API. Pharm Res 2006;23(8):1888-97.

42. Good DJ, Rodriguez-Hornedo N. Solubility advantage of pharmaceutical cocrystals. Cryst Growth Des 2009:9;225264.

43. Serajuddin ATM. Solid dispersion of poorly water-soluble drugs: early promise, subsequent problems and recent breakthroughs. J Pharm Sci 1999:88;1058-66.

44. Bethune SJ, Huang N, Jayasankar A, Rodriguez-Hornedo $\mathrm{N}$. Understanding and predicting the effect of cocrystal components and $\mathrm{pH}$ on cocrystal solubility. Cryst Growth Des 2009:9;3976-88.
45. Basavoju S, Bostrom D, Velaga SP. Indomethacin-Saccharin cocrystal: Design, synthesis and preliminary pharmaceutical characterization. Pharm Res 2008;25(3):530-41.

46. Padrela L, Rodrigues MA, Velaga SP, Fernandes AC, Matos HA, Azevedo EG. Screening for pharmaceutical cocrystals using the supercritical fluid enhanced atomization process. J Supercrit Fluids 2010;53:156-64.

47. Trask AV, Motherwell WDS, Jones W. Physical stability enhancement of theophylline via cocrystallization. Int J Pharm 2006:320;114-23.

48. Hickey MB, Peterson MI, Scoppettuolo LA, Morrisette SL, Vetter A, Guzman H, et al. Performance comparison of a new cocrystal of carbamazepine with marketed product. Eur J Pharm Biopharm 2007:67;112-9.

49. Oswald IDH, Allan DR, McGregor PA, Motherwell WDS, Parsons S, Pulham CR. The formation of paracetamol (acetaminophen) adducts with hydrogen bond acceptors. Acta Crystallogr B Struct Sci Cryst Eng Mater 2002:58;1057-66.

50. Variankaval $\mathrm{N}$, Wenslow R, Murry J, Hartman R, Helmy R, Kwong E, et al. Preparation and solid state characterization of nonstoichiometric cocrystals of a phosphodiesterase-IV inhibitor and L-tartaric acid. Cryst Growth Des 2006:6;690-700.

51. Childs SL, Rodriguez-Hornedo N, Reddy LS, Jayasankar A, Maheshwari C, McCausland L, et al. Screening strategies based on solubility and solution composition generate pharmaceutically acceptable cocrystals of carbamazepine. CrystEngComm 2008:10;856-64.

52. Trask AV, Motherwell WDS, Jones W. Pharmaceutical cocrystallization: Engineering a remedy for caffeine hydration. Cryst Growth Des 2005:5;1013-21.

53. Vangala VR, Chow PS, Tan RBH. Cocrystals and cocrystal hydrates of the antibiotic Nitrofurantoin: Structural studies and physicochemical properties. Cryst Growth Des 2012:12;5925-38.

54. Shargel L, Yu AB. Applied biopharmaceutics and pharmacokinetics. 4th ed. New York: McGraw Hill; 1999.

55. Huang Y, Zhang B, Gao Y, Zhang J, Shi L. Baicaleinnicotinamide cocrystal with enhanced solubility, dissolution and oral bioavailability. J Pharm Sci 2014:103;2330-7.

56. Cheney ML, Weyna DR, Shan N, Hanna M, Wojtas L, Zaworotko MJ. Coformer selection in pharmaceutical cocrystal development: a case study of a meloxicam aspirin cocrystal that exhibits enhanced solubility and pharmacokinetics. J Pharm Sci 2011:100;2172-81.

57. Dai XL, Li S, Chen JM, Lu TB. Improving the membrane permeability of 5-Fluorouracil via cocrystallization. Cryst Growth Des 2016:16;4430-8.

58. Sanphui P, Devi VK, Clara D, Malviya N, Ganguly S, Desiraju GR. Cocrystals of hydrochlorothiazide: Solubility and diffusion/permeability enhancements through drug-coformer interactions. Mol Pharm 2015:12;1615-22.

59. Sekhon BS. Pharmaceutical cocrystal - A review. ARS Pharmaceutica 2009:50(3);99-117.

60. NajarAA,Azim Y. Pharmaceutical cocrystals-Anew paradigm of crystal engineering. J Indian Inst Sci 2014:94(1);45-67.

61. Steed JW. The role of cocrystals in pharmaceutical design. Trends Pharmacol Sci 2013;34(3):185-93.

62. Thipparaboina R, Kumar D, Chavan RB, Shastri NR. Multidrug cocrystals: towards the development of effective therapeutic hybrids. Drug Discov Today 2016;21:481-90. 
63. Majumder M, Buckton G, Rawlinson-Malone CF, Williams AC, Spillman MJ, Pidcock E, et al. Application of hydrogenbond propensity calculations to an indomethacin-nicotinamide (1:1) cocrystal. CrystEngComm 2013:15;4041-4.

64. Cruz-Cabeza AJ. Acid-base crystalline complexes and the pKa rule. CrystEngComm 2012;14:6362-5.

65. Laszlo F. Cambridge structural database analysis of molecular complementarity in cocrystals. Cryst Growth Des 2009;9(3):1436-43.

66. Abramov YA, Loschen C, Klamt A. Rational coformer or solvent selection for pharmaceutical cocrystallization or desolvation. J Pharm Sci 2012;101(10):3687-97.

67. Mohammad AM, Amjad A, Velaga SP. Hansen solubility parameter as a tool to predict the cocrystal formation. Int $\mathrm{J}$ Pharm 2011;407:63-71.

68. Greenhalgh DJ, Williams AC, Timmins P, York P. Solubility parameters as predictors of miscibility in solid dispersions. $\mathrm{J}$ Pharm Sci 2001:88;1182-90.

69. Van Krevelen DW, Hoftyzer P. Properties of polymers, their estimation and correlation with chemical structure. $2^{\text {nd }}$ ed. Amsterdam: Elsevier Science; 1976.

70. Shete A, Murthy S, Korpale S, Yadav A, Sajane S, Sakhare $\mathrm{S}$, et al. Cocrystals of itraconazole with amino acids: screening, synthesis, solid state characterization, in vitro release and antifungal activity. J Drug Delivery SciTechnol 2015;28:46-55.

71. Musumeci D, Hunter CA, Prohens R, Scuderi S, McCabe JF. Virtual cocrystal screening. ChemSci 2011;2:883-90.

72. Yamamoto K, Tsutsumi S, Ikeda Y. Establishment of cocrystal cocktail grinding method for rational screening of pharmaceutical cocrystals. Int J Pharm 2012;437:162-71.

73. Lu E, Rodriguez-Hornedo N, Suryanarayanan R. A rapid thermal method for cocrystal screening. CrystEngComm 2008;10:665-68.

74. Zhou ZZ, Chan HM, Sung HHY, Tong HHY, Zheng Y. Identification of new cocrystal systems with stoichiometric diversity of salicylic acid using thermal method. Pharm Res 2016:33;1030-39.

75. Yamashita H, Hirakura Y, Yuda M, Terada K. Coformer screening using thermal analysis based on binary phase diagram. Pharm Res 2014;31:1946-57.

76. Lin HL, Hsu PC, Lin SY. Theophylline-citric acid co-crystals easily induced by DSC-FTIR microspectroscopy or different storage conditions. Asian J Pharm Sci 2013;8:19-27.

77. Berry DJ, Seaton CC, Clegg W, Harrington RW, Coles SJ, Horton PN, et al. Applying hot-stage microscopy to cocrystal screening: a study of nicotinamide with seven active pharmaceutical ingredients. Cryst Growth Des 2008;8(5):1697-712.

78. Blagden N, Berry DJ, Parkin A, Javed H, Ibrahim A, Gavan PT, et al. Current direction in cocrystal growth. New J Chem 2008:32;1659-72.

79. Manin AN, Voronin AP, Drozd KV, Manin NG, Bauer-Brandl A, Perlovich GL. Cocrystal screening of hydroxybenzamides with benzoic acid derivatives: a comparative study of thermal and solution based methods. Eur J Pharm Sci 2014;65:56-64.

80. Luo YH, Sun BW. Pharmaceutical cocrystals of pyrazinecarboxamide (PZA) with various carboxylic acids: crystallography, Hirshfeld surfaces and dissolution study. Cryst Growth Des 2013;13:2098-106.

81. He GW, Jacob C, Guo LF, Chow PS, Tan RBH. Screening for cocrystallization tendency: the role of intermolecular interactions. J Phys Chem 2008;112:9890-5.

82. Chun NH, Lee MJ, Song GH, Chang KY, Kim CS, Choi GJ. Combined anti-solvent and cooling method of manufacturing indomethacin-saccharin (IMC-SAC) co-crystal powders. J Cryst Growth 2014;408:112-8.

83. Zhang S, Chen H, Rasmuson AC. Thermodynamics and crystallization of a theophylline-salicylic acid cocrystal. CrystEngComm 2015; 17:4125.

84. Alhalaweh A, George S, Basavoju S, Childs SL, Rizvi SAA, Velaga SP. Pharmaceutical cocrystals of nitrofurantoin: screening, characterization and crystal structure analysis. CrystEngComm 2012;14:5078-88.

85. Karki S, Friscic T, Jones W, Motherwell WDS. Screening for pharmaceutical cocrystal hydrates via neat and liquid-assisted grinding. Mol Pharm 2007;4(3):347-54.

86. Karki S, Friscic T, Jones W. Control and interconversion of cocrystal stoichiometry in grinding: stepwise mechanism for the formation of a hydrogen-bonded cocrystal. CrystEngComm 2009;11:470-81.

87. Kotak U, Prajapati V, Solanki H, Jani G, Jha P. Cocrystallization technique its rational and recent progress. World J Pharm Pharm Sci 2015;4(4);1484-508.

88. Aher S, Dhumal R, Mahadik K, Paradkar A, York P. Ultrasound assisted cocrystallization from solution (USSC) containing a non-congruently soluble cocrystal component pair: Caffeine/ maleic acid. Eur J Pharm Sci 2010;41:597-602.

89. Yadav S, Gupta PC, Sharma N, Kumar J. Co-crystals: An alternative approach to modify physicochemical properties of drugs. Int J Pharm 2015;5(2):427-36.

90. Alhalaweh A, Velaga P. Formation of cocrystals from stoichiometric solutions of incongruently saturating systems by spray drying. Cryst Growth Des 2010;10(8):3302-5.

91. Grossjohann C, Serrano DR, Paluch KJ, O'connell P, Vellazarb L, Manesiotis P, et al. Polymorphism in sulfadimidine/4aminosalicylic acid cocrystals: solid-state characterization and physicochemical properties. J Pharm Sci 2015;104:1385-98.

92. Boksa K, Otte A, Pinal R. Matrix-assisted cocrystallization (MAC) simultaneous production and formulation of pharmaceutical cocrystals by hot-melt extrusion. J Pharm Sci 2014;103:2904-10.

93. Daurio D, Medina C, Saw R, Nagapudi K, Alvarez-Nunez F. Application of twin screw extrusion in the manufacture of cocrystals, Part-1: Four case studies. Pharmaceutics 2011;3:582-600.

94. Bhatt PM, Azim Y, Thakur TS, Desiraju GR. Cocrystals of the anti-HIV drugs lamivudine and zidovudine. Cryst Growth Des 2009;9(2):951-7.

95. Skorepova E, Husak M, Cejka J, Zamostny P, Kratochvil B. Increasing dissolution of trospium chloride by cocrystallization with urea. J Cryst Growth 2014;399:19-26.

96. Aher NS, Shinkar DM, Saudagar RB. Pharmaceutical cocrystallization: A review. J Adv Pharm Educ Res 2014;4:388-96.

97. Yadav AV, Shete AS, Dabke AP, Kulkarni PV, Sakhare SS. Cocrystals: A novel approach to modify physicochemical properties of active pharmaceutical ingredients. Indian J Pharm Sci 2009;71(4):359-70.

98. Vaghela P, Tank HM, Jalpa P. Cocrystals: A novel approach to improve the physicochemical and mechanical properties. Indo Am J Pharm Res 2014;4(10):5055-65. 
99. Mutalik S, Prambil A, Krishnan M, Achuta NU. Enhancement of dissolution rate and bioavailability of aceclofenac: A chitosan based solvent change approach. Int J Pharm 2008;350:279-90.

100. Sugandha K, Kaity S, Mukherjee S, Isaac J, Ghosh A. Solubility enhancement of ezetimibe by cocrystal engineering technique. Cryst Growth Des 2014;14:4475-86.

101. Nehm SJ, Rodriguez-Spong B, Rodríguez-Hornedo N. Phase solubility diagrams of cocrystals are explained by solubility product and solution complexation. Cryst Growth Des 2006;6:592-600.

102. Bysouth SR, Bis JA, Igo D. Cocrystallization via planetary milling: enhancing throughput of solid state screening methods. Int J Pharm 2011;411:169-71.

103. Mukaida M, Watanabe Y, Sugano K, Terada. Identification and physicochemical characterization of caffeine-citric acid cocrystal polymorphs. Eur J Pharm Sci 2015;79:61-6.

104. Shan N, Toda F, Jones W. Mechanochemistry and cocrystal formation: effect of solvent on reaction kinetics. ChemCommun 2002;2372-73.

105. Cuadra IA, Cabanas A, Cheda JAR, Martinez-Casado FJ, Pando C. Pharmaceutical cocrystals of the anti-inflammatory drug diflunisal and nicotinamide obtained using supercritical $\mathrm{CO}_{2}$ as an antisolvent. J CO2 Util 2016;13:29-37.

106. Aakeroy CB, Salmon DJ, Smith MM, Desper J. Cyanophenyloximes: reliable and versatile for hydrogen bond directed supramolecular synthesis of cocrystals. Cryst Growth Des 2006:6;1033-42.

107. Wu TK, Lin SY, Lin HL, Huang YT. Simultaneous DSCFTIR microspectroscopy used to screen and detect the cocrystal formation in real time. Bioorg Med Chem Lett 2011;21:3148-51.
108. Jiang L, Huang Y, Zhang Q, He H, Xu Y, Mei X. Preparation and solid state characterization of dapsone drug-drug cocrystals. Cryst Growth Des 2014;14:4562-73.

109. Parrott EPJ, Zeitler JA, Friscic T, Pepper M, Jones W, Day GM, et al. Testing the sensitivity of terahertz spectrocopy to changes in molecular and supramolecular structure: a study of structurally similar cocrystal. Cryst Growth Des 2009;9:145260 .

110. Stevens JS, Byard SJ, Schroeder SLM. Salt and cocrystals? Determination of protonation state by X-ray photoelectron spectroscopy (XPS). J Pharm Sci 2010;99(11):4453-7.

111. Vogt FG, Clawson JS, Strohmeier M, Edwards AJ, Pham TN, Watson SA. Solid state NMR analysis of organic cocrystals and complexes. Cryst Growth Des 2009:9;921-37.

112. EL-Gizawy SA, Osman MA, Arafa MF, El-Maghraby GM. Aerosil as a novel co-crystal co-former for improving the dissolution rate of hydrochlorothiazide. Int $\mathrm{J}$ Pharm 2015;478:773-8.

113. Li J, Liu P, Liu JP, Zhang WL, Yang JK, Fan YQ. Novel Tanshinone II A ternary solid dispersion pellets prepared by a single-step technique: In vitro and in vivo evaluation. Eur J Pharm Biopharm 2012;80(2):426-32.

114. Gurunath S, Nanjwade BK, Patila PA. Enhanced solubility and intestinal absorption of candesartan cilexetil solid dispersions using everted rat intestinal sacs. Saudi Pharm J 2014;22(3):246-57.

115. Sathali AA, Selvaraj V. Enhancement of solubility and dissolution rate of racecadotril by solid dispersion methods. $\mathrm{J}$ Curr Chem Pharm Sci 2012;2(3):209-25.

116. Fukte SR, Wagh MP, Rawat S. Coformer selection: An important tool in cocrystal formation. Int $\mathbf{J}$ Pharm Pharm Sci 2014:6;9-14. 